\title{
Mit Pregabalin gegen den zentralen Deafferenzierungsschmerz
}

Der sogenannte "central post stroke pain" (früher auch Thalamusschmerz, heute eher zentraler Deafferenzierungsschmerz genannt) ist eine Herausforderung in der Schmerztherapie. Denn verschiedene Medikamente zeigten bislang nur mäßiggradige Effektivität, häufig gelingt keine befriedigende Schmerzlinderung. Pregabalin könnte dieses Problem lösen.

$\mathrm{n}$ dieser Studie wurde das Medikament Pregabalin placebokontrolliert zur Behandlung dieses Schmerzsyndroms untersucht. Die Studie wurde über 13 Wochen durchgeführt, sie war randomisiert und beeinhaltete Dosierungen zwischen $150 \mathrm{mg}$ und $600 \mathrm{mg}$ Pregabalin täglich. Insgesamt wurden 219 Patienten eingeschlossen. Der primäre Endpunkt (Schmerzlinderung auf der numerischen Analogskala) war nicht signifikant unterschiedlich, der Wert senkte sich von 6,5 auf 4,9 unter Pregabalin und von 6,3 auf 5,0 unter Placebo. Bei einigen sekun- dären Endpunkten, wie Schlafstörungen, Angststörungen, Depression etc., zeigte sich eine signifikante bessere Wirksamkeit unter Pregabalin. Die Autoren kommen zu dem Schluss, dass trotz des Verfehlens des primären Endpunktes der Einsatz von Pregabalin beim zentralen Deafferenzierungsschmerz einigen Nutzen bietet.

Kommentar: Auch diese Studie zeigt leider, dass ein weiteres Medikament (nämlich Pregabalin) keinen signifikanten spezifischen Effekt auf den zentralen Deafferenzierungs- schmerz hat. Die Tatsache, dass einige sekundäre Endpunkte durch Pregabalin günstig beeinflusst worden sind, ist nicht verwunderlich. Insbesondere ist Pregabalin ein anxiolytisches und wahrscheinlich auch antidepressiv wirksames Medikament, so dass es natürlich zur Steigerung der Lebensqualität bei Patienten mit zentralem Deafferenzierungsschmerz beiträgt. Es bleibt abzuwarten, ob wirklich ein spezifischer Nutzen dieses Medikamentes gegeben ist. Weiterhin kann also die wirklich evidenzbasierte Therapie des zentralen Deafferenzierungsschmerzes nicht erweitert werden.

Prof. Dr. med. Stefan Evers

\section{Ist Lacosamid bei neuropathischen Schmerzen wirksam?}

Es gibt inzwischen kaum ein Antikonvulsivum mehr, das neu entwickelt worden ist und nicht auch in der Therapie von Schmerzen untersucht wurde. Hierzu gehört auch Lacosamid. In der vorliegenden Cochrane-Analyse wurde Lacosamid in Bezug auf seine Wirksamkeit gegen neuropathische Schmerzen und Fibromyalgie bei Erwachsenen untersucht - mit negativem Ergebnis.

nsgesamt konnten sechs Studien identifiziert werden, in der mit hinreichender Qualität die Wirksamkeit von Lacosamid untersucht wurde. Die Ergebnisse der Metaanalyse zeigen, dass für eine Dosierung von $200 \mathrm{mg}$ und $400 \mathrm{mg}$ Lacosamid täglich keine hinreichende Evidenz für eine Wirksamkeit vorliegt. Für eine tägliche Dosierung von 600 mg gibt es widersprüchliche Berichte im Vergleich zu Placebo. Das Medikament wurde gut vertragen, die Nebenwirkungsrate lag auf Placeboniveau. Zusammenfassend kommen die Autoren dieser Cochrane-Analyse zu dem Ergebnis, dass Lacosamid nur einen begrenzten Effekt in der Behandlung der peripheren diabetischen Neuropathie hat. Zudem wird kritisiert, dass in einigen Studien aufgrund methodischer
Probleme der Behandlungserfolg überschätzt wurden. Es ist daher wahrscheinlich, dass Lacosamid ohne sinnvollen Nutzen in der Behandlung von neuropathischen Schmerzen ist.

Kommentar: Diese Cochrane-Analyse zeigt einmal mehr, dass bei einer methodisch hochwertigen Metaanalyse von mehreren Studien unter dem Kriterium der Studienqualität ein anderes Ergebnis herauskommt, als bei der Analyse einzelner Studien. Es ist in der wissenschaftlichen Literatur der Eindruck entstanden, als ob Lacosamid in höherer Dosierung in der Behandlung von neuropathischen Schmerzen gut wirksam wäre. Die vorliegende Analyse zeigt, dass es sich häufig um methodische Mängel der Studien handelt, die zu einer Überschätzung
Kim JS, Bashford G, Murphy TK, Martin A, Dror V, Cheung R. Safety and efficacy of pregabalin in patients with central post-stroke pain. Pain 2011; 152: 1018-23

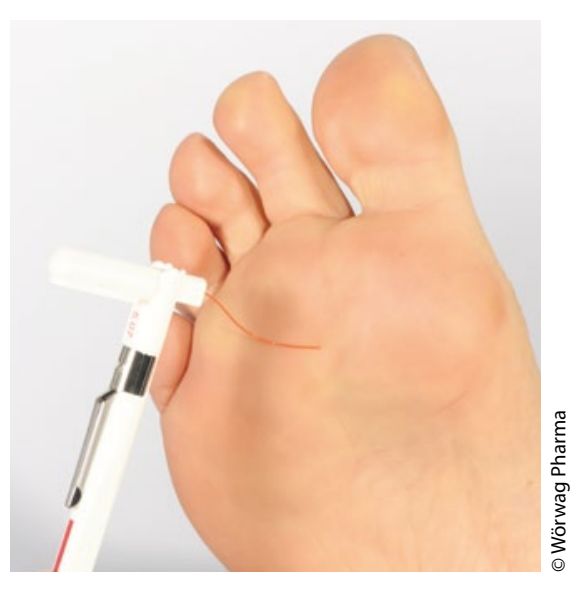

Bei neuropathischen Schmerzen und der Behandlung der peripheren diabetischen Neuropathie hat Lacosamid nur einen begrenzten Effekt.

des Effekts führen. Insgesamt gibt es keine hinreichende Evidenz für die Behandlung von neuropathischen Schmerzen mit Lacosamid.

Prof. Dr. med. Stefan Evers

Hearn L, Derry S, Moore RA. Lacosamide for neuropathic pain and fibromyalgia in adults. Cochrane Database Syst Rev 2012; 2:

CD009318 